\title{
Cotranscriptional Folding of a Bio-orthogonal Fluorescent Scaffolded RNA Origami
}

\author{
Emanuela Torelli,* Jerzy Kozyra, Ben Shirt-Ediss, Luca Piantanida, Kislon Voïtchovsky, \\ and Natalio Krasnogor*
}

Cite This: ACS Synth. Biol. 2020, 9, 1682-1692

Read Online

\section{ACCESS | Lill Metrics \& More | 回 Article Recommendations ｜（） Supporting Information}

ABSTRACT: The scaffolded origami technique is an attractive tool for engineering nucleic acid nanostructures. This paper demonstrates scaffolded RNA origami folding in vitro in which, for the first time, all components are transcribed simultaneously in a single-pot reaction. Double-stranded DNA sequences are transcribed by T7 RNA polymerase into scaffold and staple strands able to correctly fold in a high synthesis yield into the nanoribbon. Synthesis is successfully confirmed by atomic force microscopy, and the unpurified transcription reaction mixture is analyzed by an in gel-imaging assay where the transcribed RNA nanoribbons are able to capture the specific dye through the reconstituted split Broccoli aptamer showing a clear green fluorescent band. Finally, we simulate the RNA origami in silico using the nucleotide-level

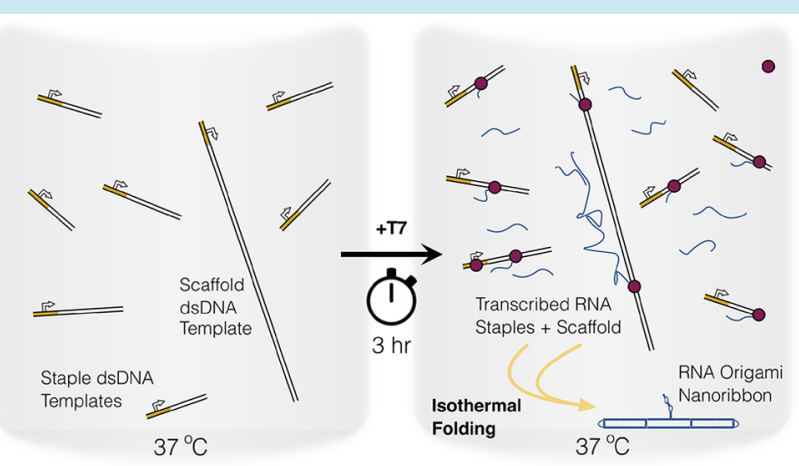
coarse-grained model oxRNA to investigate the thermodynamic stability of the assembled nanostructure in isothermal conditions over a period of time. Our work suggests that the scaffolded origami technique is a viable, and potentially more powerful, assembly alternative to the single-stranded origami technique for future in vivo applications.

KEYWORDS: cotranscriptional folding, scaffolded RNA origami, bio-orthogonal, split Broccoli aptamer, oxRNA simulation

$\mathrm{O}$ ver the past few years, the emerging field of RNA nanotechnology has progressed rapidly with demonstrations of a variety of roles and sophistication of function that RNA devices might display. These include the engineering of essential coding and noncoding functions, mRNAs, tRNAs, ribozymes, aptamers, CRISPR RNAs, etc. As a consequence, a variety of RNA nanostructures with different functionalities, sizes, and shapes have been created to investigate and successfully demonstrate their potential in nanobiomedicine and synthetic biology. ${ }^{1-12}$

Different self-assembly strategies have been adopted to design RNA nanostructures ${ }^{13}$ ranging from RNA architectonics $^{14-18}$ to single-stranded self-assembly. ${ }^{19}$

Furthermore, taking advantage of the sequential transcription reaction by bacteriophage RNA polymerase, singlestranded RNA origami has been synthesized from long ssRNA molecules. In vitro transcribed and purified RNA sequences were self-folded into hearts, rectangles, and rhombus shapes adapting the ssDNA origami design strategy and considering the helical periodicity difference between DNA B-type and RNA A-type helix. Large and complex ssRNA origami were synthesized using partially complemented double-stranded RNA and parallel crossover cohesion without limitation due to RNA kissing loop interactions. ${ }^{20}$ However, these multikilobase ssRNA nanostructures were folded using a thermal annealing ramp gradient (from 85 to $25^{\circ} \mathrm{C}$ ), thereby limiting potential in vivo applications, such as the scaffolding of enzymes. $^{21}$

On the other hand, previous work showed the synthesis of smaller ssRNA origami tiles and hexagonal lattices made by annealing and/or in vitro cotranscriptional folding that should be compatible with in vivo folding when genetically encoded and expressed in cells. ${ }^{22}$ These authors developed a strategy based on the combination of hairpins, kissing loops, and "dovetail seam" to promote and stabilize the folding during the T7 RNA polymerase in vitro transcription reaction. ${ }^{22,23}$ More recently, the ssRNA $2 \mathrm{H}$-AE-ST tile scaffold presented by Geary et al. $^{22}$ was used to create an aptamer-based FRET system where RNA tile synthesis in vivo was demonstrated by measuring FRET outputs without a direct atomic force microscopy (AFM) visualization, due to the small construct dimension. $^{24}$

Received: January 8, 2020

Published: May 29, 2020 
Li et $a l^{25}$ developed a different strategy in which the design concept was similar to the approach reported above but avoiding the use of short "dovetail seams". The RNA nanostructures were designed based on natural motifs: the folding pathway was based on hairpin formation and tertiary interactions of unpaired residues. In detail, an RNA doublesquare was designed using a 3-way loop observed in phi29 pRNA and a $90^{\circ}$-kink from hepatitis $\mathrm{C}$ virus RNA genome. These thermodynamically stable and kinetically favorable RNA nanostructures were folded both in vitro and in vivo: nonetheless, the combination of phage and viral derived structural motifs can limit the structure design, the attachment of functional units, the creation of reconfigurable and dynamic nanostructures or their suitability for future theranostic in vivo applications.

While architectonics, single-stranded self-assembly methods and single-stranded origami strategies, are well explored, scaffolded (and multistranded) RNA origami approaches are still in their infancy. Drawing from DNA origami techniques in which several short staple strands sequences promote the folding of a longer single stranded scaffold into a specific shaped structure, ${ }^{26}$ we designed and synthesized RNA origami following a similar strategy. This approach can provide several advantages such as the high synthesis yield, the possibilities to produce reconfigurable nanostructures and to incorporate multiple and different functionalities in a precise position. Indeed, previous works demonstrated the synthesis of chemically modified and siRNAs functionalized RNA origami following a thermal gradient annealing of RNA staple strands and scaffold. ${ }^{27,28}$

In our previous work, we went a step further to develop a biologically afunctional (i.e., bio-orthogonal by design) RNA origami able to fold at constant temperature $\left(37^{\circ} \mathrm{C}\right)$ after an initial denaturation step, ${ }^{29}$ which lights-up when folded to its target configuration. Seven RNA staple strands promoted the folding of a RNA bio-orthogonal synthetic De Bruijn scaffold sequence (DBS) that does not contain genetic information, restriction enzyme sites and reduces ambiguity in the addressability. ${ }^{30}$ We demonstrated the possibility to combine scaffold bio-orthogonality, physiologically compatible folding, and assembly monitoring using a new RNA-based reporter system. The folding was monitored using our new split Broccoli aptamer system. $^{29}$

Motivated by our previous study, and with the ultimate goal of enabling RNA origami expression in living cells, here we demonstrate a full isothermal protocol for scaffolded RNA origami assembly via cotranscriptional folding. We maintain the nanoribbon design simplicity and the scaffold nucleotide composition, while changing each staple in order to guarantee a reasonable yield of the desired transcripts and a low aberrant products synthesis during the in vitro transcription by T7 RNA polymerase. The RNA origami is cotranscriptionally folded into a nanoribbon shape at $37{ }^{\circ} \mathrm{C}$ : in detail, during the scaffolded RNA assembly, scaffold, and staple strands are in vitro transcribed and folded in a one-pot reaction. The RNA origami assembly is verified by gel assay and well characterized by AFM. The RNA nanostructures self-assembly is also successfully confirmed and selectively detected using our split Broccoli aptamer system: the transcription mix is analyzed by in-gel imaging and the tagged RNA origami shows a clear fluorescent band. Finally, the assembled RNA origami nanoribbon is visualized and simulated at equilibrium using the oxRNA coarse-grained model.
The in vitro transcribed and folded RNA origami described here can be compatible with expression in bacterial cells, and its self-assembly can be monitored with a protein-free fluorescence detection system using an in-gel imaging assay as a rapid and specific prescreening method.

\section{METHODS}

Materials and Reagents. (5Z)-5[(3,5-Difluoro-4hydroxyphenyl)methylene]-3,5-dihydro-2-methyl-3-(2,2,2-trifluoroethyl)-4H-imidazol-4-one (DFHBI-1T) was purchased from Tocris Biotechne. A DFHBI-1T stock solution (20 mM) was prepared in DMSO, stored in the dark at $-20{ }^{\circ} \mathrm{C}$ and used within 2 weeks. All RNA oligonucleotides were purchased from Eurogentec and resuspended in Ultra Pure distilled water to give stock solutions of $100 \mu \mathrm{M}$ and stored at $-80{ }^{\circ} \mathrm{C}$. All DNA oligonucleotides and gBlocks Gene Fragment were purchased from IDT. The DNA oligonucleotides were resuspended in Ultra Pure distilled water to give stock solutions of $100 \mu \mathrm{M}$ and stored at $-20{ }^{\circ} \mathrm{C}$. The gBlocks Gene Fragment was resuspended at a final concentration of $10 \mathrm{ng} \mu \mathrm{L}^{-1}$ and stored

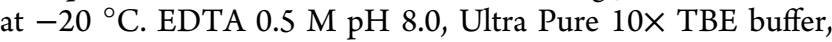
Ultra Pure $1 \mathrm{M}$ Tris- $\mathrm{HCl} \mathrm{pH}$ 7.5, Ultra Pure distilled water, 5 $\mathrm{M} \mathrm{NaCl}(0.2 \mu \mathrm{m}$ filtered), SYBR Gold, dithiothreitol molecular biology grade, GlycoBlue Coprecipitant $(15 \mathrm{mg} /$ $\mathrm{mL}$ ), inorganic pyrophosphatase, NTP (100 mM each), 6\% Novex TBE gel, 10\% Novex TBE gel and 10\% TBE-Urea gel were purchased from Thermo Fisher Scientific. Spermidine BioUltra, HEPES $1 \mathrm{M}$ pH 7 Bioreagent, $\mathrm{KCl} 1 \mathrm{M}$ BioUltra, $\mathrm{MgCl}_{2} 1 \mathrm{M}$ BioUltra, 3-aminopropyltriethoxysilane (APTES), and agarose were purchased from Sigma-Aldrich.

Scaffold and Staples Design. The synthetic RNA scaffold and RNA staples were generated with the computer code presented by Kozyra et al. ${ }^{30}$ The RNA origami ribbon (Figure S1) has been designed as previously described: ${ }^{29}$ in the present study, RNA staple strands were changed ( $\mathrm{r} 1,11, \mathrm{r} 2,12$, $\mathrm{f}$, and s1) or elongated (s2) at the $5^{\prime}$ end of few bases ( 1 up to 3 bases) in order to start with -GG or -GA (first and second nucleotides of the transcribed region) necessary for an efficient T7 transcription yield. Furthermore, all sequences were checked to reduce aberrant products synthesized during the transcription reaction. The RNA staple strands sequences and the scaffold sequence were placed downstream from the $\mathrm{T} 7$ promoter (5'-TTCTAATACGACTCACTATA-3'). The dsDNA templates for the transcription were two annealed oligonucleotides (for staple strands transcription) or a gBlocks Gene Fragment sequence (for scaffold transcription): all the dsDNA templates carried the T7 promoter sequence and the template to be transcribed (Table S1).

OxRNA Simulation. We simulated the RNA ribbon/split aptamer system using a nucleotide-level coarse-grained RNA model, oxRNA. ${ }^{31,32}$ In particular, we investigated thermodynamic stability of a preassembled nanoribbon in isothermal conditions over a period of time.

In order to simulate the system, the caDNAno design file ${ }^{33}$ were used in combination with caDNAno interface scripts (available with oxRNA software) to generate the initial topology of the simulated system. Preceding the simulation, the system was first relaxed, and the interaction type changed to RNA. To form initial configuration mutual traps were used according to the software documentation. Briefly, a harmonic force was introduced to pull selected particles from one strand of split aptamer toward reference particles on the other strand. This process used low stiffness parameter (0.1) until a 
predefined equilibrium distance of the trap was reached, in this case, 1.5 simulation units of length, corresponding roughly to the hydrogen bonding potential. A short simulation was run, and after the equilibrium was reached and the split aptamer structure formed, the mutual traps were removed.

The system was then simulated over a longer time period and the trajectory file recorded. Molecular Dynamics (MD) was the selected simulation algorithm and the simulation was run for $2 \times 10^{7}$ simulation steps which, in physical units, corresponds to $61.2 \mu \mathrm{s}$. The temperature of the system was set to $310 \mathrm{~K}\left(\sim 37{ }^{\circ} \mathrm{C}\right)$. The john thermostat, which is an Andersen-like thermostat, was used since it is the optimal thermostat that emulates Brownian dynamics. ${ }^{34}$

RNA Scaffold Synthesis and Purification. Doublestranded gBlocks Gene Fragment containing T7 promoter (gBlocks DBS scaffold, Table S1) was amplified using Phusion DNA polymerase (NEB) and DBS forward/DBS reverse primers (Table $S 1$ ), as previously described. Briefly, an initial denaturation at $98{ }^{\circ} \mathrm{C}$ for $30 \mathrm{~s}$ was followed by 15 cycles of denaturation at $98{ }^{\circ} \mathrm{C}$ for $10 \mathrm{~s}$, annealing at $60^{\circ} \mathrm{C}$ for $20 \mathrm{~s}$ and extension at $72{ }^{\circ} \mathrm{C}$ for $15 \mathrm{~s}$. Finally, an additional extension was achieved for $5 \mathrm{~min}$ at $72{ }^{\circ} \mathrm{C}$. The PCR product was purified using Monarch PCR \& DNA Cleanup kit (NEB) and the DNA concentration was measured on a NanoDrop spectrophotometer. The size of purified amplicon was evaluated on $1.5 \%$ agarose gel in TBE for $1 \mathrm{~h} 40 \mathrm{~min}$ at $110 \mathrm{~V}$ : the gel was pre stained with Nancy-520 and visualized under UV illumination. The low molecular weight DNA ladder (NEB) was used as molecular weight marker.

The purified template was transcribed in vitro at $37^{\circ} \mathrm{C}$ for 1 $\mathrm{h}$ and $30 \mathrm{~min}$ using Ampliscribe T7-Flash Transcription kit (Epicenter). After DNase treatment at $37{ }^{\circ} \mathrm{C}$ for $15 \mathrm{~min}$, the RNA transcript was purified using RNA Clean \& Concentrator (Zymo Research), quantified using a NanoDrop spectrophotometer and used as scaffold sequence for the RNA origami assembly reaction.

Alternatively, the amplified and purified scaffold template was used for cotranscriptional folding.

Conventional Folding of RNA Origami Nanoribbon. The RNA origami nanoribbon was synthesized using the conventional protocol. ${ }^{29}$ The transcribed and purified RNA scaffold was mixed with an excess of RNA staple strands in 10 $\mathrm{mM} \mathrm{MgCl} 2,20 \mathrm{mM}$ Tris-HCl pH 7.5, $1 \mathrm{mM}$ EDTA pH 8.0. ${ }^{27}$ After an initial thermal denaturation step at $75^{\circ} \mathrm{C}$ for $1 \mathrm{~min}$ and a snap cooling $\left(-1{ }^{\circ} \mathrm{C} / 0.42 \mathrm{~s}\right)$, the mixture was subjected to a folding step at $37{ }^{\circ} \mathrm{C}$ for $20 \mathrm{~min}$. Folding was performed with a SensoQuest Labcycler GeneFlow thermalcycler. Samples $(5 \mu \mathrm{L}$ of a $50 \mu \mathrm{L}$ reaction mixture) were run on $6 \%$ Novex TBE gel in $1 \times$ TBE buffer at $100 \mathrm{~V}$ for $40 \mathrm{~min}$ at low temperature (below $10^{\circ} \mathrm{C}$ ). After staining with SYBR Gold in $1 \times \mathrm{TBE}$ for $5 \mathrm{~min}$, the gels were visualized using Typhoon laser scanner and ImageQuant TL software (normal sensitivity; GE Healthcare Life Sciences). The low range ssRNA ladder (NEB) was used as molecular weight marker.

Double-Stranded DNA Template Annealing, Purification, and Transcription. RNA staples to be transcribed are less than 100 bases, ranging from 26 to 51 nucleotides. For this reason, DNA templates for in vitro transcription were obtained from two annealed single-stranded DNA oligonucleotides for each staple strand sequence: complementary sense and antisense strands (RP-HPLC or PAGE purified) containing the T7 promoter sequence and the RNA sequence to be transcribed were annealed by incubating equimolar concen- trations $(5 \mu \mathrm{M})$ of forward and reverse strand in $\mathrm{TE}$ supplemented with $12.5 \mathrm{mM} \mathrm{MgCl}_{2}(\mathrm{r} 1, \mathrm{r} 2,11$, and 12) or with $50 \mathrm{mM} \mathrm{NaCl}(\mathrm{s} 1, \mathrm{~s} 2$, and $\mathrm{f})$. The samples were heated at $95{ }^{\circ} \mathrm{C}$ for $2 \mathrm{~min}$ and slowly cooled down at $25^{\circ} \mathrm{C}(72$ cycles, $38 \mathrm{~s} /$ cycle, $-1{ }^{\circ} \mathrm{C} /$ cycle): all annealing processes were performed with a Biometra TRIO Analitik jena thermalcycler. Samples were loaded and run on $10 \%$ Novex TBE gel in $1 \times$ Tris borate EDTA (TBE) buffer at $200 \mathrm{~V}$ for $45 \mathrm{~min}$. After staining with SYBR Gold in $1 \times$ TBE for $5 \mathrm{~min}$, the gels were visualized using Typhoon laser scanner and Image Quant TL software (normal sensitivity; GE Healthcare Life Sciences). The low molecular weight DNA ladder (NEB) was used as molecular weight marker. Annealed dsDNA were purified from polyacrylamide gels by the "crush and soak" method, as previously described ${ }^{35}$ with some modifications. The bands of interest were cut out and the gel slices were transferred to a 0.5 $\mathrm{mL}$ tube that was pierced with a $20-\mathrm{G}$ needle and placed inside a $1.5 \mathrm{~mL}$ microcentrifuge tube. The tube was centrifuged at $20000 \mathrm{~g}$ for $3 \mathrm{~min}$ to force the gel through the needle hole. 0.67 $\mathrm{mL}$ of DNA soaking buffer $(0.3 \mathrm{M} \mathrm{NaCl}, 10 \mathrm{mM}$ Tris- $\mathrm{HCl} \mathrm{pH}$ 7.5, $0.97 \mathrm{mM}$ EDTA) were added to the recovered gel and incubated overnight with agitation at room temperature. Gel and soaking buffer were purified from gel debris using a Freeze'n Squeeze DNA gel extraction spin column (Bio-Rad) that was centrifuged at $20000 \mathrm{~g}$ for $3 \mathrm{~min}$ at $4{ }^{\circ} \mathrm{C}$ to recover the soaking mixture. The eluted DNA was transferred to a fresh microcentrifuge tube and precipitated at least $1 \mathrm{~h}$ at $-20{ }^{\circ} \mathrm{C}$ using $0.68 \mathrm{~mL}$ isopropanol supplemented with $1 \mu \mathrm{L}$ GlycoBlue Coprecipitant. After centrifugation at $4{ }^{\circ} \mathrm{C}(30 \mathrm{~min}$ at $20000 \mathrm{~g})$, the pellet was washed in $0.75 \mathrm{~mL}$ of $80 \%$ ice-cold ethanol, air-dried, and resuspended in $12 \mu \mathrm{L}$ of $10 \mathrm{mM}$ Tris$\mathrm{HCl} \mathrm{pH}$ 7.5. DNA samples were run on $10 \%$ Novex TBE gel and visualized as described above. Purified and unpurified samples were measured using NanoDrop One/One ${ }^{\mathrm{C}}$ spectrophotometer (Thermo Scientific).

Each purified or unpurified dsDNA template encoding the RNA staples strands were used for RNA synthesis. Transcription reactions were performed in $20 \mathrm{mM}$ Tris- $\mathrm{HCl} \mathrm{pH}$ 7.6, $10 \mathrm{mM} \mathrm{MgCl}_{2}, 2.5 \mathrm{mM}$ of each rNTPs, $4 \mathrm{mM}$ DTT, 2 $\mathrm{mM}$ spermidine, 2 units $/ \mu \mathrm{L}$ T7 RNA polymerase (NEB). The transcription mix was incubated at $37^{\circ} \mathrm{C}$ for $3 \mathrm{~h}$ and treated with $1 \mu \mathrm{L}$ DNase RNase free (NEB) at $37^{\circ} \mathrm{C}$ for $15 \mathrm{~min}$. After dilution, samples were run on $10 \%$ Novex TBE gel in $1 \times \mathrm{TBE}$ buffer at $200 \mathrm{~V}$ for $45 \mathrm{~min}$. After staining with SYBR Gold in $1 \times \mathrm{TBE}$ for $5 \mathrm{~min}$, the gels were visualized using Typhoon laser scanner and ImageQuant TL software (normal sensitivity; GE Healthcare Life Sciences). The low range ssRNA ladder (NEB) and ZR small-RNA ladder (Cambridge Bioscience) were used as molecular weight marker.

Cotranscriptional Folding of RNA Origami Nanoribbon. The concentrations of each dsDNA template encoding the RNA staples strands and scaffold were measured using NanoDrop One/One ${ }^{\mathrm{C}}$ spectrophotometer (average concentration values were calculated from 3 measurements for each sample). Different molar concentrations of DNA templates were used for RNA synthesis and RNA origami folding during the T7 transcription: $5 \mathrm{nM}$ gBlock Gene Fragment (scaffold), $5 \mathrm{nM}$ of each s1, s2, and f dsDNA, $10 \mathrm{nM}$ of each $\mathrm{r} 1$ and 12 dsDNA, $20 \mathrm{nM} 11$ dsDNA, $30 \mathrm{nM}$ r2 dsDNA. Transcription reaction was performed in $20 \mathrm{mM}$ Tris- $\mathrm{HCl} \mathrm{pH}$ 7.6, $10 \mathrm{mM} \mathrm{MgCl}_{2}, 2.5 \mathrm{mM}$ of each rNTPs, $4 \mathrm{mM} \mathrm{DTT,} 2$ $\mathrm{mM}$ spermidine, 2 units $/ \mu \mathrm{L}$ T7 RNA polymerase (NEB). The 
transcription mix was incubated at $37{ }^{\circ} \mathrm{C}$ for $3 \mathrm{~h}$ and treated with $1 \mu \mathrm{L}$ DNase RNase free (NEB) at $37^{\circ} \mathrm{C}$ for $15 \mathrm{~min}$.

Cotranscribed RNA Origami Native PAGE and In-Gel Imaging. After dilution, samples were run on $6 \%$ Novex TBE gel in $1 \times \mathrm{TBE}$ buffer at $100 \mathrm{~V}$ for $40 \mathrm{~min}$ at low temperature (below $10^{\circ} \mathrm{C}$ ). After staining with SYBR Gold in $1 \times \mathrm{TBE}$ for 5 min, the gels were visualized using Typhoon laser scanner and ImageQuant TL software (normal sensitivity; GE Healthcare Life Sciences). The low range ssRNA ladder (NEB) was used as molecular weight marker.

To confirm the RNA origami self-assembly by incorporation of Split Broccoli aptamer system, in-gel imaging with fluorophore DFHBI-1T ${ }^{36}$ was performed with some modifications. Briefly, RNA origami sample, partially folded samples and Broccoli aptamer (prepared as previously described $^{29}$ ) were loaded in the polyacrylamide gels. The gels were washed three times for $5 \mathrm{~min}$ in RNase free water and then stained for 20-25 min in aptamer buffer containing $1.26 \mu \mathrm{M}$ DFHBI-1T, $40 \mathrm{mM}$ HEPES $\mathrm{pH}$ 7.4, $100 \mathrm{mM} \mathrm{KCl}, 1$ $\mathrm{mM} \mathrm{MgCl}{ }_{2}$. The gels were imaged using Typhoon laser scanner (excitation $488 \mathrm{~nm}$, emission $532 \mathrm{~nm}$ ): bands were analyzed using ImageQuant TL software. Then, the gels were washed three times with Ultra Pure distilled water, stained with SYBR Gold in $1 \times$ TBE for $8 \mathrm{~min}$, and visualized using Typhoon laser scanner. The low range ssRNA ladder (NEB) was used as molecular weight marker.

Atomic Force Microscope (AFM) Imaging. All the experiments were conducted on a commercial AFM Cypher ES (Asylum Research, Oxford Instruments, Santa Barbara, CA) and with the tip and cantilever fully immersed into the liquid. The vertical oscillation of the tip was controlled by photothermal excitation (Blue Drive) and the experiments were conducted at $25.0 \pm 0.1{ }^{\circ} \mathrm{C}$. All measurements were conducted in Amplitude Modulation (AM-AFM) mode using SCANASYST-Fluid+ (Bruker, Camarillo, CA) cantilever and with a set point ratio between the free amplitude and imaging amplitude of $\sim 0.8$.

Freshly cleaved mica was passivated for $5 \mathrm{~min}$ with $10 \mu \mathrm{L}$ of $0.1 \%$ APTES in water to ensure the adhesion of the negative charged RNA origami structures on the negative mica surface. After three washing steps with $10 \mathrm{mM} \mathrm{MgCl}, 20 \mathrm{mM}$ Tris$\mathrm{HCl} \mathrm{pH} 7.5,1 \mathrm{mM}$ EDTA $\mathrm{pH}$ 8.0, the transcription reactions were diluted $1: 100$ in the same buffer, added $(2 \mu \mathrm{L})$ to the passivated mica surface, allowed to adsorb for $5 \mathrm{~min}$ in a chamber and imaged immediately. When purified RNA origami samples were imaged, $8 \mu \mathrm{L}$ of sample were added to the passivated mica surface and allowed to adsorb for $5 \mathrm{~min}$ in a chamber as above.

All the images were corrected for tilt (line or plane fattening) and lightly low-pass filtered to remove grainy noise using the WSxM software (Nanotec Electronica, Madrid, Spain). ${ }^{37}$

\section{RESULTS AND DISCUSSION}

Staple Strands and RNA Origami Design. Recently, a small functional scaffolded RNA origami made from just few sequences demonstrated gene knockdown functionality. It was a RNA nanostructure synthesized from a short mRNA fragment scaffold and nine synthetic RNA strands. ${ }^{28}$

Here, our aim is to understand if scaffolded RNA origami, decorated with a split Broccoli aptamer, could be made by in vitro cotranscriptional folding. More precisely, we investigate the cotranscriptional folding of both the scaffold itself and its staples, which has not yet been explored. As a proof of principle, we decided to design a simple RNA origami that involves seven staple strands and a scaffold sequence. Our approach uses all the components as products of the transcription machinery rather than of chemical synthesis. Moreover RNA self-assembly occurs during the transcription reaction. With the ultimate future goal to construct a "biocircuit board" to spatially organize biomolecules and thus regulate metabolic activity, a simple ribbon nanostructure has been chosen and designed. The design includes the precise organization of a split aptamer system that can allow to monitor the assembly inside a living cell without using fluorescent reporter proteins and thus reducing the energetic burden to the cells.

Recently, we designed a 212 nt biologically inert (i.e., bioorthogonal) and uniquely addressable De Bruijn scaffold sequence (DBS) characterized by lack of genetic information, restriction enzyme sites, and reduced ambiguity in the addressability: the bio-orthogonality of the RNA scaffold designed in silico was demonstrated in E. coli cells. ${ }^{29}$

Here, we used our previous optimized RNA scaffold and a new set of seven staple strands, starting with -GG or -GA nucleotides at the $5^{\prime}$ end (Figure S1 and Table S1) in order to promote a reasonable transcriptional yield and allow an efficient control of the $5^{\prime}$ sequence content. ${ }^{38}$ In detail, transcription reactions of each RNA sequence were carried out with T7 RNA polymerase and synthetic DNA containing the T7 promoter: the above-mentioned $5^{\prime}$ end starting nucleotides can guarantee efficient transcription and efficient control of the $5^{\prime}$ sequence content during the T7 polymerase reaction. ${ }^{38}$

Furthermore, although T7 RNA polymerase is a highly specific enzyme, the desired short RNA is usually accompanied by undesired products that are longer or shorter than the expected transcript. ${ }^{39-44}$ Besides its higher DNA affinity, T7 RNA polymerase is able to synthesize RNA from single or double-stranded RNA template. ${ }^{42,43}$ It has been demonstrated that RNA extension can occur if the $3^{\prime}$ end has a selfcomplementarity (trans mechanism) or folds back on itself in cis to form extendible duplexes. ${ }^{40,42,43}$ Next generation RNASeq analysis has demonstrated that primer extension occurs predominantly via a cis self-primer mechanism and strongly depends on pairing $A$ at the $3^{\prime}$ end with the $U, 9$ bases upstream; ${ }^{43}$ as a result, partially double-stranded RNA byproducts are synthesized. In order to reduce the amount of longer undesired RNA extension products catalyzed by $\mathrm{T} 7$ RNA polymerase, all staple strands sequences were examined to take account of the $3^{\prime}$ end self-complementarity and pairing between the $3^{\prime}$ end adenine and uracil, 9 bases upstream; the analysis revealed that all RNA sequences were lacking of these distinct $3^{\prime}$ end characteristics.

In addition, false transcriptions are mainly observed when the correct product is free of stable secondary structures at the 3 ' end: the aberrant transcripts are longer than the coded RNA. $^{40}$ It has been reported that improved transcription of the correct length RNA can be obtained by adding hairpins to the $3^{\prime}$ and 5 ' ends; these hairpins decrease the transcription of incorrect-length products, but still a series of bands can remain. ${ }^{45,46}$ In our work, all the sequences were optimized to weaken secondary structures and avoid hairpin formation. ${ }^{30}$

DNA Template Preparation and Transcription. The scaffold sequence and each of the staple strands sequences were placed downstream from the T7 RNA polymerase promoter and the linear double-stranded DNA templates were 


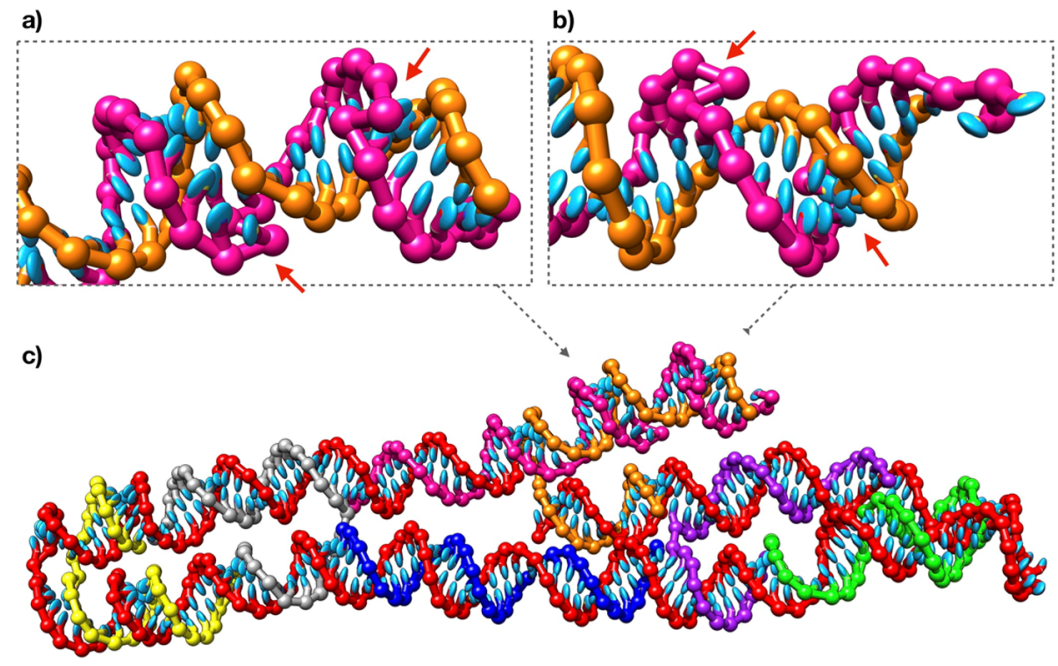

Figure 1. oxRNA simulation of a preassembled RNA ribbon. Final configuration of the simulated system is shown after an equilibration period. The RNA ribbon is formed of the scaffold (red), which is bound by 5 staples (various colors). The two split aptamer strands (pink and orange) are located at the $5^{\prime}$ and $3^{\prime}$ ends of the scaffold, accordingly. The aptamer structure forming over the noncontinuous section of top duplex is flexible: it can either stack with the left or the right duplex, or it can remain in an unstacked conformation. Enlarged views show the front (a) and the back (b) of the aptamer structure in respect to the RNA ribbon (c). Red arrows indicate the location of bulge loops.

characterized by blunt ends in order to reduce the synthesis of spurious transcripts.

Scaffold DNA template was purified from an amplified gBlocks Gene Fragment (Figure S2). As staple strands to be transcribed were shorter (ranging from 26 to 51 bases), DNA templates were obtained from an annealing step of two RPHPLC or PAGE purified oligonucleotides.

Annealed DNA templates corresponding to staple strands showed multiple bands (Figure S3) presumably due also to the presence of byproducts in addition to the desired chemically synthesized oligonucleotide (RP-HPLC and PAGE purification usually can provide a purity level up to $85 \%$ and around $85-$ $90 \%$, respectively).

To increase the purity, annealed DNA templates were purified from polyacrylamide gel by the "crush and soak" method using GlycoBlue as coprecipitant to facilitate the DNA recovery and reach a concentration compatible with the subsequent transcription reaction avoiding any mixture dilution. All samples were run and checked on native PAGE (Figure S4).

The use of GlycoBlue coprecipitant at final concentrations $(22 \mu \mathrm{g} / \mathrm{mL})$ below the recommended value $(50-150 \mu \mathrm{g} / \mathrm{mL})$ did not allow an accurate DNA quantification due to the blue dye absorbance and the low A260/A230 ratio value (0.8). Furthermore, there were no differences in the transcription products when purified and unpurified dsDNA samples were used as template. For these reasons, we decided to in vitro transcribe unpurified DNA templates.

Each RNA sequence was synthesized during T7 polymerase in vitro transcription from dsDNA templates. T7 polymerase is usually produced by expressing the RNA polymerase gene of bacteriophage $\mathrm{T} 7$ in $E$. coli cells and is used to study transcription mechanism in vitro or as a convenient approach to synthesize RNA strands. Once the enzyme is isolated from E. coli cultures for in vitro reactions, the TRIS working buffer composition is optimized considering the transcriptional activity of the enzyme in vitro and the specific application. The in vitro transcription system is affected by stimulatory natural polyamines, particularly spermidine, and requires freshly prepared dithiothreitol (DTT). The polyamine additive can activate $\mathrm{T} 7$ polymerase reaction and increases the fidelity of RNA transcripts: the activation effect is concentrationdependent in a wide range. ${ }^{47}$ The transcription TRIS buffer requires also magnesium ions and usually free ions concentration is adjusted according to the nucleotide concentration. $^{38}$ Finally, in the enzymatic reaction, RNA polymerase produces inorganic pyrophosphate (PPi) as byproduct: PPi released by the reaction binds magnesium ions to form $\mathrm{Mg}_{2} \mathrm{PPi}$, which can influence the RNA synthesis. ${ }^{48}$ Inorganic pyrophosphatase catalyzes the hydrolysis of the pyrophosphate to two orthophosphates and can be used to improve the yield of the in vitro transcription reaction.

Considering all the above-mentioned factors (magnesium ions, spermidine, DTT, and inorganic pyrophosphatase), the RNA origami folding buffer composition, ${ }^{27,29}$ and in order to improve the transcription of each dsDNA template, different DTT and spermidine concentrations were tested with or without inorganic pyrophosphatase. In detail, the initial folding buffer $\left(10 \mathrm{mM} \mathrm{MgCl}_{2}, 20 \mathrm{mM}\right.$ Tris- $\mathrm{HCl} \mathrm{pH}$ 7.5, $1 \mathrm{mM}$ EDTA $\mathrm{pH} 8.0)^{27,29}$ was supplemented with $2.5 \mathrm{mM}$ of each rNTPs, while EDTA was removed to avoid inhibition of the DNase treatment performed at the end of the transcription reaction. Then the transcription buffer was supplemented with (i) 10 $\mathrm{mM}$ DTT and $2 \mathrm{mM}$ spermidine; ${ }^{19}$ (ii) $10 \mathrm{mM}$ DTT, $2 \mathrm{mM}$ spermidine $^{19}$ and $0.2 \mathrm{U}$ inorganic pyrophosphatase; (iii) $4 \mathrm{mM}$ DTT and $2 \mathrm{mM}$ spermidine; or (iv) $4 \mathrm{mM}$ DTT and $0.2 \mathrm{U}$ inorganic pyrophosphatase.

In vitro transcribed RNA sequences were analyzed by denaturing PAGE. The transcription of each staple strand dsDNA template in $4 \mathrm{mM}$ DTT and $2 \mathrm{mM}$ spermidine showed a distinct main band corresponding to the full-length transcript (Figure S5). For this reason we decided to use the same concentrations for scaffold dsDNA template transcription: the transcribed scaffold resulted in a main band of the expected size (Figure S6). In addition to the full-length transcript, the synthesis of a pattern of products with a higher molecular weight was also observed as previously described. ${ }^{40,46}$ TrianaAlonso et al. ${ }^{40}$ demonstrated the synthesis of longer transcripts 
as a secondary process following an induction time that is different for every template: a minimal concentration of transcript is required for aberrant transcripts that accumulated at the end of long incubations as a result of a late event during the transcription. As RNA staple strands were designed to not support RNA-primed RNA extension, we hypothesized that, after the production of a certain amount of transcript, T7 polymerase accepted template RNA sequences that were not folded in $3^{\prime}$ end stable secondary structures. We then assumed that during the cotranscriptional folding, duplex formation related to the RNA origami folding can sequester the staple strands $3^{\prime}$ end reducing RNA-templated extension and undesired byproducts.

Finally, abortive synthesis was observed in T7 RNA polymerase transcription as a fundamental and major feature of early stages: several short transcripts were produced (maximum length of $12 \mathrm{nt}$ ) due to a kinetic competition between dissociation of the enzyme-DNA-RNA complex and incorporation of successive nucleotides. ${ }^{49}$ During the scaffold and staple strands transcription, a low amount of abortive products (Figure S5 and Figure S6) were produced without influencing the transcription efficiency. Previous studies showed that both abortive products and intermediate products were observed as an unavoidable result of the in vitro transcription. ${ }^{41,49}$

OxRNA Simulation. The simulated RNA ribbon/split aptamer system stayed at equilibrium and the split aptamer structure was preserved at $37^{\circ} \mathrm{C}$. The two bulge loops ( $3 \mathrm{nt}$ and 2 nt-long, respectively) were visible in the aptamer structure (Figure 1, red arrows). The hybridization between split aptamer strands and the origami scaffold allowed for a degree of flexibility between the aptamer and the ribbon. Interestingly, the simulation suggested that the internal loop (located between the red arrows) formed a double-helical structure despite the mismatched nucleotides. However, the Gibbs's free energy of that interaction was higher than a typical complementary sequence of similar length and thus less stable.

RNA Origami Cotranscriptional Folding and AFM Imaging. Self-assembly at physiologically compatible temperatures can expand and improve the RNA nanotechnology applications under intracellular conditions. To date, the ability to design RNA nanostructures that cotranscriptionally fold into the target shape has been demonstrated using different approaches. ${ }^{19,22,50,51}$

As a proof of principle, here we demonstrate the cotranscriptional folding of a genetically encoded scaffolded RNA origami using our previously designed bio-orthogonal DBS scaffold. All double-stranded DNA templates encoding scaffold and seven staple strands were mixed in a one-pot reaction and used to transcribe RNA sequences able to fold into the desired nanostructure. The cotranscriptional folding was performed at $37^{\circ} \mathrm{C}$ for $3 \mathrm{~h}$ using T7 RNA polymerase in the transcriptional buffer containing $4 \mathrm{mM}$ DTT and $2 \mathrm{mM}$ spermidine. Furthermore, two partially folded RNA scaffold samples were transcribed from dsDNA template encoding respectively: (i) scaffold, Staple s1 and s2; (ii) Staple s1, s2, 11, and $\mathrm{r} 1$. These samples were considered to demonstrate not only their different electrophoretic migration patterns, but also their different fluorescence band intensities in the following ingel imaging experiments.

After a DNase treatment to digest template dsDNA, the unpurified transcription mixtures were directly analyzed by nondenaturing polyacrylamide gel electrophoresis: the gel image showed a distinct band around the expected size (black arrow in Figure 2) with a different migration distance

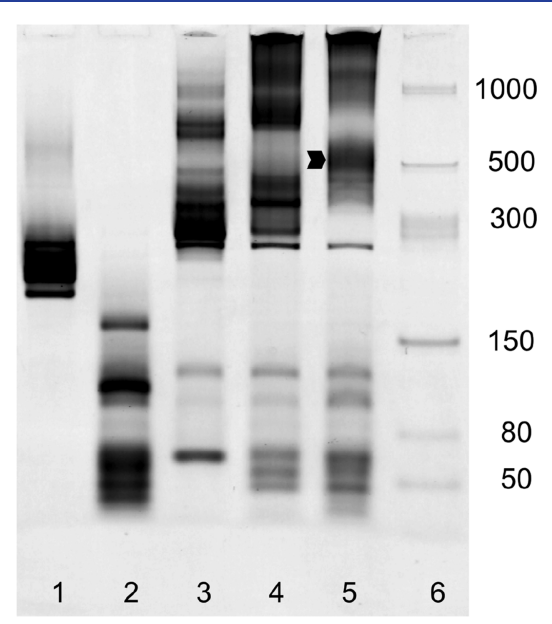

Figure 2. 6\% TBE gel electrophoresis of transcription products after SYBR Gold staining. Lanes. (1) transcribed RNA scaffold; (2) transcribed RNA staple strands; (3) transcribed RNA scaffold, Staples s1 and s2; (4) transcribed RNA scaffold and RNA Staples s1, s2, r1, and 11; (5) cotranscriptional folded RNA (black arrow); (6) low range ssRNA ladder. Molecular size in nucleotides are indicated.

compared to that of partially folded products, suggesting the correct folding. Bands corresponding to scaffold and partially folded products were almost not present in the RNA origami sample. The origami yield percentage was estimated from native polyacrylamide $\mathrm{gel}^{25}$ and was approximately $20 \%$ of all in vitro transcribed RNA.

In order to compare cotranscriptional and conventional prepared RNA nanostructures, RNA origami was also synthesized from transcribed and purified RNA scaffold and RNA staple strands, and used as control. After an initial thermal denaturation, the sample solution was subjected to a folding step at $37{ }^{\circ} \mathrm{C}$ for $20 \mathrm{~min}$ and verified by gel electrophoresis (Figure S7) as previously described. ${ }^{29}$

The cotranscriptional folded nanostructure band showed a slight difference in the migration distance (Figure 2) when compared to conventional assembled nanostructure (Figure S7), as previously observed: ${ }^{19}$ the electrophoretic migration of unpurified transcribed samples was influenced by the different and more complex reaction mixture composition. ${ }^{52}$

Finally, we found a higher band closed to the well (Figure 2): as a result of both thermal ramp or cotranscriptional folding, higher bands can appear ${ }^{19,22,28,53}$ and can correspond to aggregates, ${ }^{28}$ spurious assemblies ${ }^{53}$ or undesired kinetically trapped products. $^{22}$

In detail, abortive and elongated RNA products of incorrect length are an unavoidable result of the in vitro transcription ${ }^{41,49}$ and they can contribute to the formation of spurious assemblies. ${ }^{53}$ Stewart et al. ${ }^{53}$ showed that despite the presence of these transcription byproducts able to generate unknown folding, the one-pot method yields the desired assemblies. The latter authors showed examples of gel electrophoresis images with a significant amount of abortive and elongated products. Furthermore, AFM images of tubular assemblies and flat lattices revealed a "noisy" environment. In the perspective of future in vivo isothermal folding, it should be noted that aberrant products produced in vitro by $\mathrm{T} 7 \mathrm{RNA}$ polymerase are not commonly synthesized inside living cells. ${ }^{40}$ 

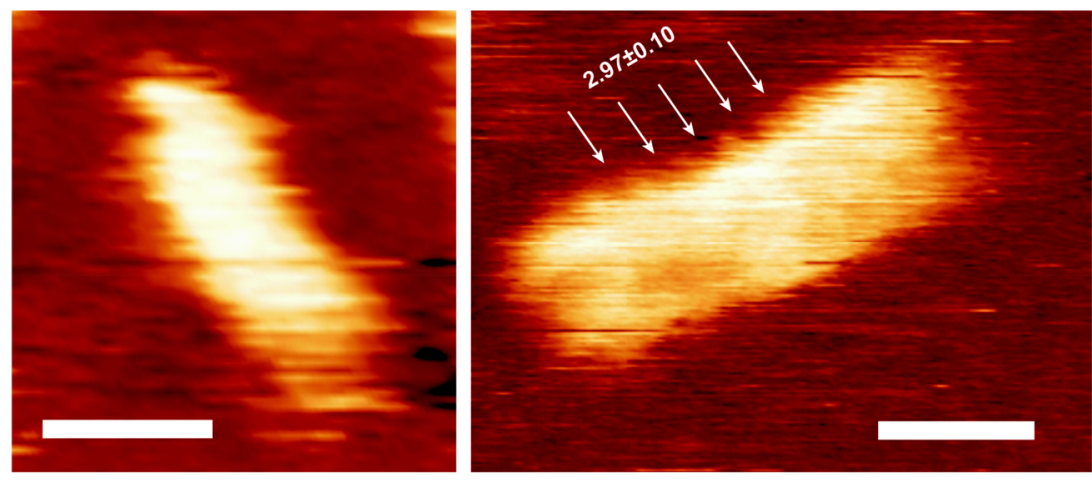

0

$3 \mathrm{~nm}$

Figure 3. High-resolution AFM images of cotranscriptional folded RNA origami. The images were taken in solution immediately after cotranscription. The white arrows show the dsRNA helical pitch $\left(3.2 \pm 0.3 \mathrm{~nm}^{54}\right)$. Scale bar: $10 \mathrm{~nm}$.

The unpurified RNA origami sample was characterized by AFM immediately after the cotranscriptional folding. Samples were diluted and deposited on a passivated mica surface using a $0.1 \%$ APTES solution in water instead of $\mathrm{Mg}^{2+}$. Indeed, it has been suggested that the different phosphate groups' orientation in dsRNA compared to dsDNA can be a possible reason for the difficult dsRNA adsorption on mica using magnesium ions. ${ }^{54}$ The estimated RNA nanostructures' dimensions were approximately $27 \mathrm{~nm} \times 5 \mathrm{~nm}$, as the A-form RNA helix revealed a rise per base pair of $0.28 \mathrm{~nm} .{ }^{54}$ AFM images confirmed the correct folding of the cotranscribed RNA nanostructures with average lengths of $27.2 \pm 3.5 \mathrm{~nm}$ and 8.4 $\pm 1.8 \mathrm{~nm}$ (Figure 3 and Figure S8), calculated considering shape closed and consistent with the design, as previously reported. ${ }^{25}$ Cotranscribed RNA origami images were compatible to that of purified conventional RNA origami sample (Figure 4 and Figure S8) previously well characterized, ${ }^{29}$

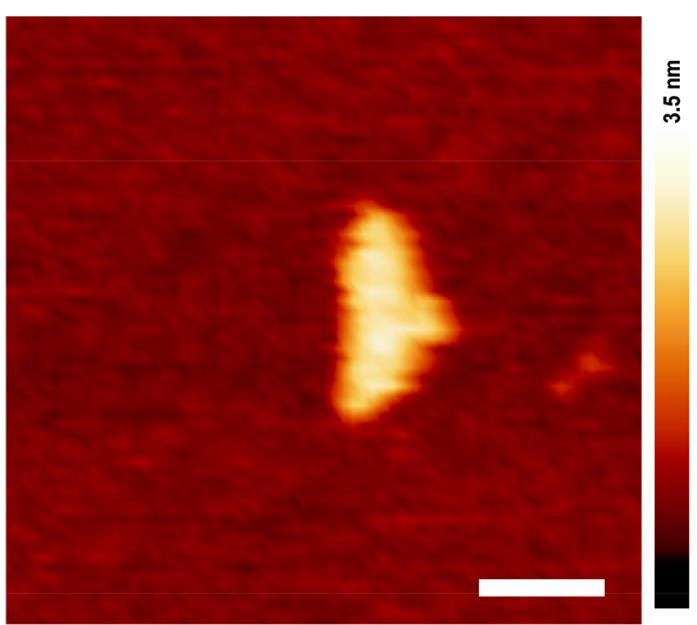

Figure 4. High-resolution AFM image of conventional folded RNA origami assembled from transcribed and purified RNA scaffold and RNA staple strands. Scale bar: $20 \mathrm{~nm}$.

indicating that the RNA scaffold and staple strands folded into the desired shape also during the transcription reaction. RNA nanostructures showed a double-stranded RNA mean periodicity of $3.1 \pm 0.3 \mathrm{~nm}$ (Figure 3, right): this measurement was consistent with the A-form helical pitch of dsRNA previously reported. ${ }^{54}$ The split Broccoli aptamer was sometimes visible as a slightly elevated side on the origami surface (Figure 3, right) or as a thin protrusion (Figure 4 and Figure S9) depending on the orientation during the deposition on the mica surface: the aptamer can either stack with the left or the right duplex, and also be out of plane and not necessarily always in the position pictured in Figure 1. It has been already noted that not all the modifications of the hexagonal minilattices with aptamers are well visible by AFM imaging due to the extension orientation with respect to the scanning direction. $^{55}$

Interactions between RNA nanostructures were also observed in AFM images (Figure S9), presumably due to interaction between left ( $5^{\prime}$-UGUUAUACAG-3') and right $\left(5^{\prime}\right.$-CCGGUCCGGC-3') ssRNA scaffold regions, as previously noted. $^{29}$

Furthermore, agglomerates were imaged during AFM scanning (Figure S9) and this was consistent with the higher band visible near the gel well (Figure 2). As we discussed above, the formation of undesired assembly can be promoted by runoff elongated RNA molecules produced during the transcription process, as recently described. ${ }^{53}$ Finally, smaller structures were imaged: it should be noted that RNA nanostructures can be damaged during deposition on mica and by AFM tip, resulting in artificial structure. Previous studies underlined that structural heterogeneity can be observed as the result of an unavoidable fragmentation during sample deposition and imaging. ${ }^{25,56}$

Light-up RNA Origami and In-Gel Imaging. Light-up RNA aptamers have been selected to induce a fluorescence emission upon binding specific small molecules allowing a protein-free RNA tagging. ${ }^{57}$ Since their development, fluorescent aptamers have been used in vitro and in vivo as a valid alternative to other imaging strategies (e.g., fluorescent in situ hybridization and molecular beacons $\left.{ }^{57,58}\right)$. Malachite green, Mango, Spinach, and Broccoli aptamers have been inserted in the design of different RNA nanostructures to successfully demonstrate the self-assembly and folding through their functional activation and fluorescence emission. ${ }^{9,24,29,55}$ Considering characteristics such as the short size, the robust folding under physiological conditions, and the specificity for a noncytotoxic and cell permeable dye, Broccoli aptamer ${ }^{59}$ was recently chosen to functionalize an RNA origami nanoribbon. ${ }^{29}$ The Broccoli aptamer was divided into two nonfunctional halves each of which was elongated in the $5^{\prime}$ or $3^{\prime}$ end with two sequences complementary to the bioorthogonal RNA scaffold. When the RNA origami self- 

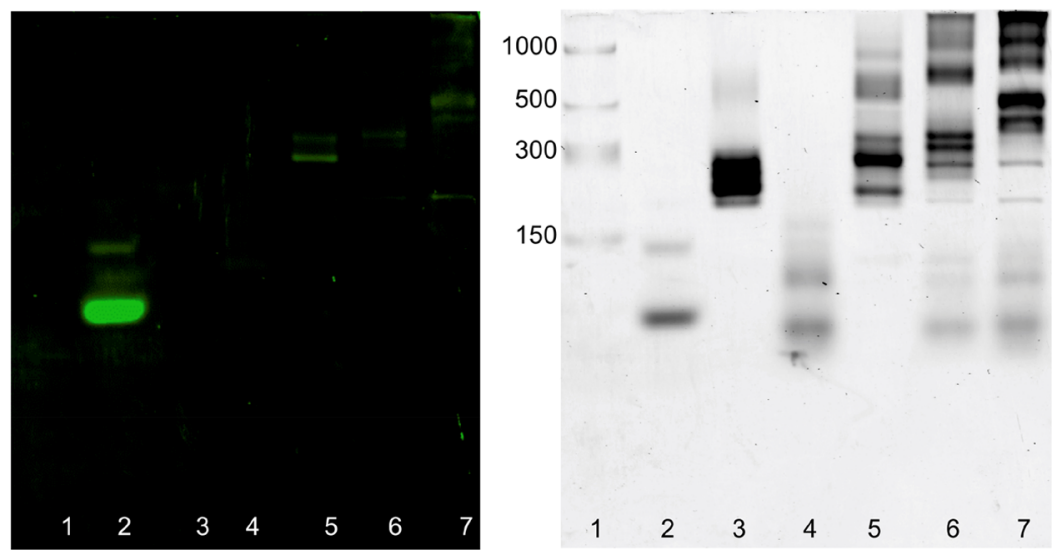

Figure 5. In-gel imaging of cotranscriptional folded light-up RNA origami. 10\% TBE gel electrophoresis after DFHBI-1T (left) and after SYBR Gold (right) staining. The gel was stained with DFHBI-1T for $25 \mathrm{~min}$ to visualize Broccoli aptamer (positive control) and cotranscriptional folded RNA origami. After 3 washing steps, the gel was stained with SYBR Gold for 5 min to detect transcribed RNA. Lanes: (1) low range ssRNA ladder; (2) Broccoli aptamer; (3) transcribed RNA scaffold; (4) transcribed RNA staples; (5) transcribed scaffold, s1 and s2 staples; (6) transcribed scaffold, s1, s2, 11, and r1 staples; (7) transcribed RNA origami. Molecular sizes in nucleotides are indicated.

assembled, the split sequences, called Split s1 and Split s2, were in closed proximity turning on the fluorescence. ${ }^{29}$

Here, after cotranscriptional folding, unpurified partially assembled scaffold and RNA origami samples were diluted, resolved by PAGE, and analyzed by in-gel imaging in order to monitor the self-assembly well-characterized by AFM imaging. In detail, after PAGE and three washing steps, the gel was stained with DFHBI-1T, which selectively binds Broccoli aptamer. $^{36}$ The DFHBI-1T concentration $(1.26 \mu \mathrm{M})$ and the staining time $(20-25 \mathrm{~min})$ were optimized in order to reduce undesired fluorescent background related to the loading of unpurified transcribed samples instead of samples purified and concentrated through ultrafiltration. ${ }^{29}$ Fluorescent imaging of the gel showed a prominent bright band with the largest peak area (approximately 6 times higher than each of the two lower bands, lane 7 in Figure 5) corresponding to the well folded RNA nanostructures band revealed by UV imaging after SYBR Gold staining. Lanes 5 and 6 (Figure 5, left) showed partially folded scaffold with low fluorescent bands which were not present in lane 7 corresponding to the full assembled origami. Lane 7 (Figure 5, left) showed a faint fluorescent band with higher mobility compared to the RNA scaffold. In the case of conventional folded RNA origami sample, this band was not visible. ${ }^{29}$ We concluded that in the in vitro transcription case, the faint fluorescent band corresponded to unknown transcribed sequences hybridized to the scaffold: indeed, transcription byproducts able to generate unknown folding are an unavoidable result of the in vitro transcription. ${ }^{53}$

The double staining allowed us to confirm the correct assembly considering both the specific migration distance and the fluorescence of the RNA nanostructures band.

Considering our result and the selective rapid in-gel imaging of Broccoli tagged RNA expressed in E. coli, ${ }^{36}$ we concluded that this simple analysis system can be used as a prescreening method to monitor and check if genetically encoded nanostructures are expressed in bacterial cells.

\section{CONCLUSIONS}

Our future aim is focused on the in vivo synthesis of 2D RNA origami that can act as a "bio-circuit board" allowing the precise molecular arrangement through protruding oligonucleotides. The resulting spatial organization can represent a strategy to control metabolic processes in engineered bacterial cells. ${ }^{21}$ Toward this perspective, we present a cotranscriptionally folded RNA origami as a further step along this direction.

In the context of in vivo origami expression to precisely control biomolecular processes, our aim is not focused on an efficient way to produce complex RNA nanostructures in large scale. Indeed, in vivo expression of RNA assembly should not impose a cost to the host cell consuming excessive resources for expression and interfering with endogenous molecules. ${ }^{60,61}$ Nonetheless, several factors can be considered and evaluated when a high-yield production of more complex nanostructures is investigated during in vitro transcription. For example the transcription incubation time, $\left[\mathrm{Mg}^{2+}\right],\left[\mathrm{Mn}^{2+}\right],[\mathrm{DTT}]$, $[\mathrm{NTP}]$, and Tritox-X100 addition represent few parameters that can be tested. ${ }^{39,62,63}$

We have demonstrated the cotranscriptional folding of a bioorthogonal scaffolded RNA origami in a one-pot reaction, revealing that the self-assembly of scaffold and staple strands can occur also in the transcription reaction mixture at $37{ }^{\circ} \mathrm{C}$ from double-stranded templates. In alternative to other strategies previously reported (i.e., single-stranded origami ${ }^{22}$ and strands self-assembly ${ }^{19}$ ), the scaffolded RNA origami technique can be successfully used to design and synthesize a desired nanostructure at constant, physiologically compatible temperature, overcoming the use of synthetic purified RNA sequences not possible in living cells.

Split Broccoli aptamer functionalization was introduced into the RNA origami design allowing a simple and specific screening of the well folded nanostructures by in-gel imaging using a cell permeable and compatible dye. This approach can further suggest and confirm the use of our split light-up aptamer $^{29}$ as a reporter system to monitor the folding, avoiding the use of fluorescent proteins. Furthermore, the fluorogenic aptamer can be replaced with other functional sequences required for different purposes.

In conclusion, our results represent a further step toward in vivo self-assembly of nucleic acid nanostructures, and expand the design strategies used to synthesize nanostructures tailored to specific applications. Future works can be focused on demonstrating that more complex nanostructures self-assemble by the same cotranscriptional method. 
In a wider picture, genetically encoded scaffolded RNA origami could represent a pathway to construct a nanostructure able to direct specific cell metabolic pathways through an orthogonal spatial and post transcriptional control.

\section{ASSOCIATED CONTENT}

\section{S) Supporting Information}

The Supporting Information is available free of charge at https://pubs.acs.org/doi/10.1021/acssynbio.0c00009.

Details about the sequences, design of the cotranscriptional folded RNA origami nanoribbon, gel images, and AFM images (PDF)

\section{AUTHOR INFORMATION}

\section{Corresponding Authors}

Emanuela Torelli - Interdisciplinary Computing and Complex BioSystems (ICOS), Centre for Synthetic Biology and Bioeconomy (CSBB), Devonshire Building, Newcastle University, Newcastle upon Tyne NE1 7RX, United Kingdom; 다이. orcid.org/0000-0002-3313-1088;

Email: emanuela.torelli@newcastle.ac.uk

Natalio Krasnogor - Interdisciplinary Computing and Complex BioSystems (ICOS), Centre for Synthetic Biology and Bioeconomy (CSBB), Devonshire Building, Newcastle University, Newcastle upon Tyne NE1 7RX, United Kingdom; ○ orcid.org/0000-0002-2651-4320;

Email: natalio.krasnogor@newcastle.ac.uk

\section{Authors}

Jerzy Kozyra - Interdisciplinary Computing and Complex BioSystems (ICOS), Centre for Synthetic Biology and Bioeconomy (CSBB), Devonshire Building, Newcastle University, Newcastle upon Tyne NE1 7RX, United Kingdom

Ben Shirt-Ediss - Interdisciplinary Computing and Complex BioSystems (ICOS), Centre for Synthetic Biology and Bioeconomy (CSBB), Devonshire Building, Newcastle University, Newcastle upon Tyne NE1 7RX, United Kingdom

Luca Piantanida - Department of Physics, Durham University, Durham DH1 3LE, United Kingdom

Kislon Voïtchovsky - Department of Physics, Durham University, Durham DH1 3LE, United Kingdom; $\odot$ orcid.org/ 0000-0001-7760-4732

Complete contact information is available at:

https://pubs.acs.org/10.1021/acssynbio.0c00009

\section{Author Contributions}

The manuscript was written by N.K. and E.T. Sequence design and oxRNA simulation were performed by J.W.K. B.S.E. conducted sequence analysis. AFM imaging was conducted by L.P.; all images were discussed with E.T. All other experiments were conceived by N.K. and E.T. All authors have given approval to the final version of the manuscript.

\section{Notes}

The authors declare no competing financial interest.

\section{ACKNOWLEDGMENTS}

This work was supported by Engineering and Physical Sciences Research Council Grant EP/N031962/1. Prof. Krasnogor is supported by a Royal Academy of Engineering Chair in Emerging Technology award. K.V. and L.P. acknowledge funding from the Biotechnology and Biological Sciences
Research Council (Grant BB/M024830/1). N.K. secured funding.

\section{REFERENCES}

(1) Guo, P. (2010) The emerging field of RNA nanotechnology. Nat. Nanotechnol. 5, 833-842.

(2) Shukla, G. C., Haque, F., Tor, Y., Wilhelmsson, L. M., Toulmé, J. J., Isambert, H., Guo, P., Rossi, J. J., Tenenbaum, S. A., and Shapiro, B. A. A. (2011) Boost for the Emerging Field of RNA Nanotechnology. ACS Nano 5, 3405-3418.

(3) Afonin, A. K., Kasprzak, W. K., Bindewald, E., Kireeva, M., Viard, M., Kashlev, M., and Shapiro, B. A. (2014) In silico Design and Enzymatic Synthesis of Functional RNA Nanoparticles. Acc. Chem. Res. 47, 1731-174.

(4) Afonin, K. A., Viard, M., Kagiampakis, I., Case, C. L., Dobrovolskaia, M. A., Hofmann, J., Vrzak, A., Kireeva, M., Kasprzak, W. K., KewalRamani, V. N., and Shapiro, B. A. (2015) Triggering of RNA Interference with RNA-RNA, RNA-DNA, and DNA-RNA Nanoparticles. ACS Nano 9, 251-259.

(5) Li, H., Lee, T., Dziubla, T., Pi, F., Guo, S., Xu, J., Li, C., Haque, F., Liang, X. J., and Guo, P. (2015) RNA as a stable polymer to build controllable and defined nanostructures for material and biomedical applications. Nano Today 10, 631-655.

(6) Kim, H., Park, Y., Kim, J., Jeong, J., Han, S., Lee, J. S., and Lee, J. B. (2016) Nucleic Acid Engineering: RNA following the trail of DNA. ACS Comb. Sci. 18, 87-89.

(7) Jasinski, D., Haque, F., Binzel, D. W., and Guo, P. (2017) Advancement of the Emerging Field of RNA Nanotechnology. ACS Nano 11, 1142-1164.

(8) Haque, F., Pi, F., Zhao, Z., Gu, S., Hu, H., Yu, H., and Guo, P. (2018) RNA versatility, flexibility, and thermostability for practice in RNA nanotechnology and biomedical applications. WIREs RNA 9, No. e1452.

(9) Liu, J., Wang, Z., Zhao, S., and Ding, B. (2018) Multifunctional nucleic acid nanostructures for gene therapies. Nano Res. 11, 50175027.

(10) Xu, C., Li, H., Zhang, K., Binzel, D. W., Yin, H., Chiu, W., and Guo, P. (2019) Photo-controlled release of paclitaxel and model drugs from RNA pyramids. Nano Res. 12, 41-48.

(11) Li, H., Wang, S., Ji, Z., Xu, C., Shlyakhtenko, L. S., and Guo, P. (2019) Construction of RNA nanotubes. Nano Res. 12, 1952-1958.

(12) Ohno, H., Akamine, S., and Saito, H. (2019) RNA nanostructures and scaffolds for biotechnology applications. Curr. Opin. Biotechnol. 58, 53-61.

(13) Grabow, W. W., and Jaeger, L. (2014) RNA Self-Assembly and RNA Nanotechnology. Acc. Chem. Res. 47, 1871-1880.

(14) Shu, D., Moll, W. D., Deng, Z., Mao, C., and Guo, P. (2004) Bottom-up assembly of RNA arrays and superstructures as potential parts in nanotechnology. Nano Lett. 4, 1717-1723.

(15) Chworos, A., Severcan, I., Koyfman, A. Y., Weinkam, P., Oroudjev, E., Hansma, H. G., and Jaeger, L. (2004) Building programmable jigsaw puzzles with RNA. Science 306, 2068-2072.

(16) Severcan, I., Geary, C., Chworos, A., Voss, N., Jacovetty, E., and Jaeger, L. (2010) A polyhedron made of tRNAs. Nat. Chem. 2, 772779 .

(17) Shu, Y., Cinier, M., Shu, D., and Guo, P. (2011) Assembly of multifunctional $\phi 29$ pRNA nanoparticles for specific delivery of siRNA and other therapeutics to targeted cells. Methods 54, 204-214.

(18) Ishikawa, J., Furuta, H., and Ikawa, Y. (2013) RNA tectonics (tectoRNA) for RNA nanostructure design and its application in synthetic biology. Wiley Interdiscip. Rev.: RNA 4, 651-664.

(19) Afonin, A. K., Bindewald, E., Yaghoubian, A. J., Voss, N., Jacovetty, E., Shapiro, B. A., and Jaeger, L. (2010) In vitro assembly of cubic RNA-based scaffolds designed in silico. Nat. Nanotechnol. 5, $676-682$

(20) Han, D., Qi, X., Myhrvold, C., Wang, B., Dai, M., Jiang, S., Bates, M., Liu, Y., An, B., Zhang, F., Yan, H., and Yin, P. (2017) Single-stranded DNA and RNA origami. Science 358, 1402. 
(21) Delebecque, C. J., Lindner, A. B., Silver, P. A., and Aldaye, F. A. (2011) Organization of intracellular reactions with rationally designed RNA assemblies. Science 333, 470-474.

(22) Geary, C., Rothemund, P. W. K., and Andersen, E. S. (2014) A single-stranded architecture for cotranscriptional folding of RNA nanostructures. Science 345, 799-804.

(23) Sparvath, S. L., Geary, C. W., and Andersen, E. S. (2017) Computer-Aided Design of RNA Origami Structures. In 3D DNA Nanostructure, Method and Protocols, Springer Protocols (Ke, Y., and Wang, P., Eds.), pp 51-80, Humana Press, New York.

(24) Jepsen, M. D. E., Sparvath, S. M., Nielsen, T. B., Langvad, A. H., Grossi, G., Gothelf, K. V., and Andersen, E. S. (2018) Development of a genetically encodable FRET system using fluorescent RNA aptamers. Nat. Commun. 9, 18.

(25) Li, M., Zheng, M., Wu, S., Tian, C., Liu, D., Weizmann, Y., Jiang, W., Wang, G., and Mao, C. (2018) In vivo production of RNA nanostructures via programmed folding of single-stranded RNAs. Nat. Commun. 9, 2196.

(26) Rothemund, P. W. K. (2006) Folding DNA to create nanoscale shapes and patterns. Nature 440, 297-302.

(27) Endo, M., Takeuchi, Y., Emura, T., Hidaka, K., and Sugiyama, H. (2014) Preparation of Chemically Modified RNA Origami Nanostructures. Chem. - Eur. J. 20, 15330-15333.

(28) Høiberg, H. C., Sparvath, S. M., Andersen, V. L., Kjems, J., and Andersen, E. S. (2019) An RNA origami Octahedron with Intrinsic siRNA for Potent Gene Knockdown. Biotechnol. J. 14, 1700634.

(29) Torelli, E., Kozyra, J. W., Gu, J. Y., Stimming, U., Piantanida, L., Voitchovsky, K., and Krasnogor, N. (2018) Isothermal folding of a light-up bio-orthogonal RNA origami nanoribbon. Sci. Rep. 8, 6989.

(30) Kozyra, J., Ceccarelli, A., Torelli, E., Lopiccolo, A., Gu, J. Y., Fellermann, H., Stimming, U., and Krasnogor, N. (2017) Designing Uniquely Addressable Bio-orthogonal Synthetic Scaffolds for DNA and RNA Origami. ACS Synth. Biol. 6, 1140-1149.

(31) Sulc, P., Romano, F., Ouldridge, T. E., Doye, J. P. K., and Louis, A. A. (2014) A nucleotide-level coarse-grained model of RNA. J. Chem. Phys. 140, 235102.

(32) Matek, C., Sulc, P., Randisi, F., Doye, J. P. K., and Louis, A. A. (2015) Coarse-grained modelling of supercoiled RNA. J. Chem. Phys. $143,243122$.

(33) Douglas, S. M., Marblestone, A. H., Teerapittayanon, S., Vazquez, A., Church, G. M., and Shih, W. M. (2009) Rapid prototyping of 3D DNA-origami shapes with caDNAno. Nucleic Acids Res. 37, 5001-5006.

(34) Andersen, H. C. (1980) Molecular dynamics simulations at constant pressure and/or temperature. J. Chem. Phys. 72, 2384-2393.

(35) Churchman, L. S., and Weissman, J. S. (2012) Native Elongating Transcript Sequencing (NET-seq). Curr. Protoc. Mol. Biol. 98, 14.4.1-14.4.17.

(36) Filonov, G. S., Kam, C. W., Song, W., and Jaffrey, S. R. (2015) In-gel imaging of RNA processing using Broccoli reveals optimal aptamer expression strategies. Chem. Biol. 22, 649-660.

(37) Horcas, I., Fernandez, R., Gomez-Rodriguez, J. M., Colchero, J., Gomez-Herrero, J., and Baro, A. M. (2007) WSXM: a software for scanning probe microscopy and a tool for nanotechnology. Rev. Sci. Instrum. 78, No. 013705.

(38) Beckert, B., and Masquida, B. (2011) Synthesis of RNA by In Vitro Transcription. In RNA. Methods in Molecular Biology (Methods and Protocols) (Nielsen, H., Ed.), pp 29-41, Humana Press, New York.

(39) Milligan, J. F., Groebe, D. R., Witherell, G. W., and Uhlenbeck, O. C. (1987) Oligoribonucleotide synthesis using T7 RNA polymerase and synthetic DNA templates. Nucleic Acids Res. 15, 8783-8798.

(40) Triana-Alonso, F. J., Dabrowski, M., Wadzack, J., and Nierhaus, K. H. (1995) Self-coded 3'-Extension of Run-off Transcripts Produces Aberrant Products during in Vitro Transcription with T7 RNA Polymerase. J. Biol. Chem. 270, 6298-6307.

(41) Arnaud-Barbe, N., Cheynet-Sauvion, V., Oriol, G., Mandrand, B., and Mallet, F. (1998) Transcription of RNA templates by T7 RNA polymerase. Nucleic Acids Res. 26, 3550-3554.
(42) Nacheva, G. A., and Berzal-Herranz, A. (2003) Preventing nondesired RNA-primed RNA extension catalyzed by T7 RNA polymerase. Eur. J. Biochem. 270, 1458-1465.

(43) Gholamalipour, Y., Mudiyanselage, A. K., and Martin, C. T. (2018) $3^{\prime}$ end additions by T7 RNA polymerase are RNA selftemplated, distributive and diverse in character-RNA-Seq analyses. Nucleic Acids Res. 46, 9253-9263.

(44) Gholamalipour, Y., Johnson, W. C., and Martin, C. T. (2019) Efficient inhibition of RNA self-primed extension by addition of competing 3'-capture DNA-improved RNA synthesis by T7 RNA polymerase. Nucleic Acids Res. 47, No. e118.

(45) Kim, J., White, K. S., and Winfree, E. (2006) Construction of an in vitro bistable circuit from synthetic transcriptional switches. Mol. Syst. Biol. 2, 68.

(46) Oesinghaus, L., and Simmel, F. C. (2019) Switching the activity of Cas12a using guide RNA strand displacement circuits. Nat. Commun. 10, 2092.

(47) Iwata, M., Izawa, M., Sasaki, N., Nagumo, Y., Sasabe, H., and Hayashizaki, Y. (2000) T7 Polymerase Activation and Improvement of the Transcriptional Sequencing by Polyamines. Bioorg. Med. Chem. $8,2185-2194$.

(48) Akama, S., Yamamura, M., and Kigawa, T. (2012) A Multiphysics Model of In Vitro Transcription Coupling Enzymatic Reaction and Precipitation Formation. Biophys. J. 102, 221-230.

(49) Martin, C. T., Muller, D. K., and Coleman, J. E. (1988) Processivity in Early Stages of Transcription by T7 RNA polymerase. Biochemistry 27, 3966-3974.

(50) Afonin, A. K., Kireeva, M., Grabow, W. W., Kashlev, M., Jaeger, L., and Shapiro, B. A. (2012) Co-transcriptional Assembly of Chemically Modified RNA Nanoparticles Functionalized with siRNA. Nano Lett. 12, 5192-5195.

(51) Yu, J., Liu, Z., Jiang, W., Wang, G., and Mao, C. (2015) De novo design of an RNA tile that self-assembles into a homo-octameric nanoprism. Nat. Commun. 6, 5724.

(52) Shihabi, Z. K. (2004) Effect of sample composition on electrophoretic migration. Application to hemoglobin analysis by capillary electrophoresis and agarose electrophoresis. J. Chromatogr. A 1027, 179-184.

(53) Stewart, J. M., Subramanian, H. K. K., and Franco, E. (2017) Self-Assembly of multi-stranded RNA motifs into lattices and tubular structures. Nucleic Acids Res. 45, 5449-5457.

(54) Ares, P., Fuentes-Perez, M. E., Herrero-Galán, E., Valpuesta, J. M., Gil, A., Gomez-Herrero, J., and Moreno-Herrero, F. (2016) High resolution atomic force microscopy of double-stranded RNA. Nanoscale 8, 11818-11826.

(55) Chopra, A., Sagredo, S., Grossi, G., Andersen, E. S., and Simmel, F. C. (2019) Out-of-Plane Aptamer Functionalization of RNA Three-Helix Tiles. Nanomaterials 9, 507.

(56) Wei, B., Dai, M., and Yin, P. (2012) Complex shapes selfassembled from single-stranded DNA tiles. Nature 485, 623-626.

(57) Ouellet, J. (2016) RNA Fluorescence with Light-Up Aptamers. Front. Chem. 4, 29.

(58) Chandler, M., Lyalina, T., Halman, J., Rackley, L., Lee, L., Dang, D., Ke, W., Sajja, S., Woods, S., Acharya, S., Baumgarten, E., Christopher, J., Elshalia, E., Hrebien, G., Kublank, K., Saleh, S., Stallings, B., Tafere, M., Striplin, C., and Afonin, K. A. (2018) Broccoli Fluorets: Split Aptamers as a User-Friendly Fluorescent Toolkit for Dynamic RNA Nanotechnology. Molecules 23, 3178.

(59) Filonov, G. S., Moon, J. D., Svensen, N., and Jaffrey, S. R. (2014) Broccoli: Rapid Selection of an RNA Mimic of Green Fluorescent Protein by Fluorescence-Based Selection and Directed Evolution. J. Am. Chem. Soc. 136, 16299-16308.

(60) Rodrigo, G., Prakash, S., Shen, S., Majer, E., Daròs, J. A., and Jaramillo, A. (2017) Model-based design of RNA hybridization networks implemented in living cells. Nucleic Acids Res. 45, 97979808.

(61) Li, J., Green, A. A., Yan, H., and Fan, C. (2017) Engineering nucleic acid structures for programmable molecular circuitry and intracellular biocomputation. Nat. Chem. 9, 1056-1067. 
(62) Afonin, K. A., Kireeva, M., Grabow, W. W., Kashlev, M., Jaeger, L., and Shapiro, B. A. (2012) Co-transcriptional assembly of Chemically Modified RNA Nanoparticles Functionalized with siRNAs. Nano Lett. 12, 5192-5195.

(63) Kanwal, F., Chen, T., Zhang, Y., Simair, A., Rujie, C., Sadaf Zaidi, N. U. S., Guo, X., Wei, X., Siegel, G., and Lu, C. (2018) LargeScale in Vitro Transcription, RNA Purification and Chemical Probing Analysis. Cell. Physiol. Biochem. 48, 1915-1927. 